\title{
Complications, Costs, and Quality Outcomes of Patients Undergoing Cervical Deformity Surgery with Intraoperative BMP Use
}

\author{
Varshneya, Kunal ; Wadhwa, Harsh ; Pendharkar, Arjun V ; Medress, Zachary A ; Stienen, Martin N ;
} Ratliff, John K ; Veeravagu, Anand

\begin{abstract}
STUDY DESIGN An epidemiological study using national administrative data from the MarketScan database. OBJECTIVE To identify the impact of bone morphogenetic protein (BMP) on postoperative outcomes in patients undergoing adult cervical deformity (ACD) surgery. SUMMARY OF BACKGROUND DATA BMP has been shown to stimulate bone growth and improve fusion rates in spine surgery. However, the impact of BMP on reoperation rates and postoperative complication rate is controversial. METHODS We queried the MarketScan database to identify patients who underwent ACD surgery from 2007-2015. Patients were stratified by BMP use in the index operation. Patients under 18 and those with any history of tumor or trauma were excluded. Baseline demographics and comorbidities, postoperative complication rates and reoperation rates were analyzed. RESULTS A total of 13,549 patients underwent primary ACD surgery, of which 1155 (8.5\%) had intraoperative BMP use. The overall 90-day complication rate was $27.6 \%$ in the non-BMP cohort and $31.1 \%$ in the BMP cohort $(\mathrm{p}<0.05)$. Patients in the BMP cohort had longer average length of stay (4.0 days vs 3.7 days, $\mathrm{p}<$ $0.05)$ but lower revision surgery rates at 90 -days $(14.5 \%$ vs $28.3 \%, \mathrm{p}<0.05), 6$ months $(14.9 \%$ vs $28.6 \%$, $\mathrm{p}<0.05)$, 1 year $(15.7 \%$ vs $29.2 \%, \mathrm{p}<0.05)$, and 2 years $(16.5 \%$ vs $29.9 \%, \mathrm{p}<0.05)$ postoperatively. BMP use was associated with higher payments throughout the 2-year follow-up period (107, 975vs97,620, $\mathrm{p}<0.05)$. When controlling for baseline group differences, BMP use independently increased the odds of postoperative complication (OR 1.22, 95\% CI 1.1 - 1.4) and reduced the odds of reoperation throughout 2-years of follow-up (OR 0.49, 95\% CI 0.4 - 0.6). CONCLUSIONS Intraoperative BMP use has benefits for fusion integrity in ACD surgery but is associated with increased postoperative complication rate. Spine surgeons should weigh these benefits and drawbacks to identify optimal candidates for BMP use in ACD surgery. LEVEL OF EVIDENCE 3.
\end{abstract}

DOI: https://doi.org/10.1097/BRS.0000000000003629

Posted at the Zurich Open Repository and Archive, University of Zurich

ZORA URL: https://doi.org/10.5167/uzh-189178

Journal Article

Published Version

Originally published at:

Varshneya, Kunal; Wadhwa, Harsh; Pendharkar, Arjun V; Medress, Zachary A; Stienen, Martin N; Ratliff, John K; Veeravagu, Anand (2020). Complications, Costs, and Quality Outcomes of Patients Undergoing Cervical Deformity Surgery with Intraoperative BMP Use. Spine, 45(22):1553-1558.

DOI: https://doi.org/10.1097/BRS.0000000000003629 
SPINE An International Journal for the study of the spine, Publish Ahead of Print

DOI: $10.1097 / B R S .0000000000003629$

\section{Complications, Costs, and Quality Outcomes of Patients Undergoing Cervical Deformity Surgery with Intraoperative BMP Use}

Kunal Varshneya BS, ${ }^{1}$ Harsh Wadhwa BS, ${ }^{1}$ Arjun V. Pendharkar MD, ${ }^{1}$ Zachary A. Medress MD, ${ }^{1}$ Martin N. Stienen MD/FEBNS, ${ }^{1,2}$ John K. Ratliff MD, ${ }^{1}$ Anand Veeravagu MD ${ }^{1}$

${ }^{1}$ Neurosurgery AI Lab \& Department of Neurosurgery, Stanford University School of Medicine, Stanford, CA

${ }^{2}$ Department of Neurosurgery, University Hospital Zurich and Clinical Neuroscience Center, University of Zurich, Zurich, Switzerland

\section{orresponding author:}

Anand Veeravagu, MD

Assistant Professor of Neurosurgery

Stanford University School of Medicine

300 Pasteur Drive

Stanford, CA 94305

Phone: (650)723-0320

Email: anandv2@stanford.edu

The manuscript submitted does not contain information about medical device(s)/drug(s).

No funds were received in support of this work.

Relevant financial activities outside the submitted work: grants.

Copyright @ 2020 Wolters Kluwer Health, Inc. Unauthorized reproduction of this article is prohibited. 


\begin{abstract}
Study Design: An epidemiological study using national administrative data from the MarketScan database.
\end{abstract}

Objective: To identify the impact of bone morphogenetic protein (BMP) on postoperative outcomes in patients undergoing adult cervical deformity (ACD) surgery.

Summary of Background Data: BMP has been shown to stimulate bone growth and improve fusion rates in spine surgery. However, the impact of BMP on reoperation rates and postoperative complication rate is controversial.

Methods: We queried the MarketScan database to identify patients who underwent ACD surgery from 2007-2015. Patients were stratified by BMP use in the index operation. Patients under 18 and those with any history of tumor or trauma were excluded. Baseline demographics and comorbidities, postoperative complication rates and reoperation rates were analyzed.

Results: A total of 13,549 patients underwent primary ACD surgery, of which $1155(8.5 \%)$ had intraoperative BMP use. The overall 90-day complication rate was $27.6 \%$ in the nonBMP cohort and $31.1 \%$ in the BMP cohort $(\mathrm{p}<0.05)$. Patients in the BMP cohort had longer average length of stay (4.0 days vs 3.7 days, $\mathrm{p}<0.05$ ) but lower revision surgery rates at 90days $(14.5 \%$ vs $28.3 \%, \mathrm{p}<0.05), 6$ months $(14.9 \%$ vs $28.6 \%$, p < 0.05$), 1$ year $(15.7 \%$ vs $29.2 \%, \mathrm{p}<0.05)$, and 2 years $(16.5 \%$ vs $29.9 \%, \mathrm{p}<0.05)$ postoperatively. BMP use was associated with higher payments throughout the 2-year follow-up period $(\$ 107,975$ vs $\$ 97,620, \mathrm{p}<0.05)$. When controlling for baseline group differences, BMP use independently increased the odds of postoperative complication (OR 1.22, 95\% CI $1.1-1.4$ ) and reduced the odds of reoperation throughout 2-years of follow-up (OR 0.49, 95\% CI $0.4-0.6$ ).

Conclusions: Intraoperative BMP use has benefits for fusion integrity in ACD surgery but is associated with increased postoperative complication rate. Spine surgeons should weigh these benefits and drawbacks to identify optimal candidates for BMP use in ACD surgery.

Key Words: cervical deformity; ACD; BMP; Marketscan; outcomes

\title{
Level of Evidence: 3
}




\section{Key Points}

- Patients with BMP use for ACD surgery have longer length of stay, but lower rates of revision surgery

- BMP use was associated with higher payments throughout the 2-year follow-up period

- Though BMP use was associated with increased odds for developing a postoperative complication, it reduced the odds for reoperation at all time points throughout 2-years of follow-up

- Intraoperative BMP use increases fusion integrity, but these benefits must be weighed with the drawbacks

\section{Introduction}

Cervical spinal deformity can have a significant impact on patient health related quality of life (HRQOL). ${ }^{1}$ Though many recent publications have described the impact of corrective surgery on patients with deformities of the thoracolumbar spine, the literature surrounding cervical re-alignment is sparse. ${ }^{2}$ High postoperative complication rates, implant failure, and need for revision remain significant concerns after these operations, with age, number of levels within the fusion construct, and baseline comorbidities having been identified as important predictors of increased postoperative morbidity. ${ }^{3-6}$ As such, quality improvement efforts must focus on identifying clinical strategies that may improve both short- and long-term surgical outcomes.

Bone morphogenetic protein 2 (BMP-2) was first identified by Marshall Urist in 1965 as a potent osteo-inductive agent. ${ }^{7}$ Food and Drug Administration (FDA) approval of rhBMP2 in 2002 for single-level anterior lumbar interbody fusion (ALIF) led to an increase in its onand off-label use for various spinal fusion procedures. ${ }^{8-13}$ Indeed, following studies demonstrated that BMP may reduce operative time and length of stay in patients undergoing spine surgery. ${ }^{10,14-18}$ However, there have been studies suggesting an increased risk of postoperative complications with the use of BMP. ${ }^{19-22}$

BMP use in spinal deformity surgery remains controversial, with studies indicating that rates of reoperation are similar with and without BMP. ${ }^{23,24}$ However, a 2015 study 
utilizing a statewide administrative dataset found a 7.5 -fold decrease in reoperations due to pseudoarthrosis with the use of BMP intraoperatively. ${ }^{25}$ The debate regarding the risks and benefits of BMP are ongoing and the practice of individual surgeons differs considerably with regards to its utilization. Thus, we aimed to utilize a national claims dataset and provide insight into the impact of intraoperative BMP on postoperative complications, reoperations, and costs in patients undergoing cervical spinal deformity surgery.

\section{Methods}

\section{Data Source}

This study obtained a sample of the MarketScan Commercial Claims and Encounters database (Truven Health Analytics, Ann Arbor, MI) from 1/1/2007 to 12/31/2016. This database is a collection of commercial inpatient, outpatient, and pharmaceutical claims of more than 75 million employees, retirees, and dependents representing a substantial portion 
18 years of age, with a history of tumor or trauma, or who underwent single-level fusions were excluded.

\section{Variables and Outcomes}

Patients were stratified into two cohorts based on the presence (BMP group) or absence of a BMP procedure code on the index deformity surgery date (no BMP group; control), and these cohorts were mutually exclusive. Individual demographic information and comorbidity status including history of diabetes, congestive heart failure (CHF), arterial hypertension, cardiac arrhythmia, liver disease, osteoporosis, and tobacco and alcohol use of each patient were gathered. Surgical approach type (anterior, posterior, or combined) was also noted (Table 1).

The primary outcome of this study was the rate of reoperation following index surgery. This was measured at 90-days, 6 months, 1 year, and 2 years following admission for ACD surgery. A revision surgery was defined by the presence of the aforementioned CPT codes for deformity surgery after the index stay for the ACD surgery. A secondary outcome measure was the presence of a postoperative complication (defined as a complication occurring within 90-days of the index deformity surgery). These included pulmonary complications, acuteposthemorrhagic anemia, sepsis, central nervous system, cardiac, renal, or wound complications, deep vein thrombosis (DVT), pulmonary embolism (PE), acute kidney injury (AKI) or dysphagia (Table 2, Table 3).

Healthcare utilization data were also collected. Payments (stratified into physician, hospital \& total payments of the index hospitalization, as well as 90-day, 6-month, 1 year, and 2 years total payments) and 90-day readmission rates were included (Table 3). An analysis of the BMP use over time in anteriorly corrected deformity patients was also conducted (Figure 1).

To isolate the impact of BMP on outcomes, a multivariate regression analysis was conducted with all significantly different baseline covariates (approach type, tobacco use). Outcomes of this analysis, with respect to complications and reoperation rate (Table 4). Two sample t-tests or chi-squared tests were used as appropriate. $P$ values were interpreted as significant if $\mathrm{p}<$ 0.05. Information about the level of significance is provided in each table caption. Statistical analysis was conducted in R Studio version 1.0.153. 


\section{Ethical considerations}

All data from these databases are de-identified, and thus this study is exempt from IRB approval in accordance with the Health Insurance Portability and Accountability Act of 1996.

\section{Results}

\section{Patient Cohort}

A total of 13,549 patients underwent primary ACD surgery, of which 1155 (8.5\%) had intraoperative BMP use. Patients who received an BMP were similarly aged (BMP: 52.1 years, non-BMP: 51.5 years, $\mathrm{p}=0.0710$ ) and in both cohorts, approximately two-thirds of patients were female. Baseline rates of CHF, hypertension, diabetes, and osteoporosis were similar between cohorts. Tobacco use was higher in the BMP cohort $(33.4 \%$ vs $29.0 \%$, p < 0.05). Anterior surgical approach was preferred in both cohorts, however, rates of posterior and combined surgery differed $(\mathrm{p}<0.05)$ (Table 1).

\section{Complications}

The overall 90-day complication rate significantly varied between cohorts $(P<0.05)$. The complication rate was $27.6 \%$ in the non-BMP cohort and $31.1 \%$ in the BMP cohort. The specific adverse events driving this effect were deficiency and posthemorrhagic anemia, occurring $1.3 \mathrm{x}$ and $1.23 \mathrm{x}$ more frequently after BMP use ( $\mathrm{p}$ 0.05). Rates of dysphagia, DVT, PE, and wound complications were similar between the two cohorts. (Table 2).

\section{Quality and health care utilization outcomes}

Patients in whose ACD procedures BMP was used had longer lengths of stay (4.0 days vs 3.7 days, $\mathrm{p}<0.05)$. The BMP cohort had significantly lower rates of revision surgeries at 90 days (14.5\% vs $28.3 \%, \mathrm{p}<0.05), 6$ months ( $14.9 \%$ vs $28.6 \%$, p < 0.05$), 1$ year $(15.7 \%$ vs $29.2 \%$, $\mathrm{p}<0.05)$, and 2 years $(16.5 \%$ vs $29.9 \%, \mathrm{p}<0.05)$ following the index surgery. Costs of index hospitalization were significantly higher in the BMP cohort $(\$ 91,488$ vs $\$ 80,211, p<0.05)$. Hospital payments were individually higher in the BMP cohort $(\mathrm{p}<0.05)$; however, physician payments were similar between BMP and non-BMP patients. Including payments 
after discharge, BMP use in patients was associated with higher payments throughout the 2year follow-up period $(\$ 107,975$ vs $\$ 97,620, p<0.05)$ (Table 3).

After controlling for baseline covariates (approach type, tobacco use) in multivariate regression, BMP use independently increased the odds for developing a postoperative complication (OR 1.22, 95\% CI $1.1-1.4$ ). BMP use also reduced the odds for a reoperation at all time points throughout the 2 years of follow-up (OR 0.49, 95\% CI $0.4-0.6$ ).

The use of BMP during anterior cervical deformity correction decreased over the study period. In 2007, 13.5\% of patients who underwent ACD surgery received BMP, whereas by 2015 , the number was $8.5 \%$. This measures to a $-37.0 \%$ decrease in BMP use over the study period in anterior cervical deformity surgery with a particularly prominent drop in the year of 2008 (Figure 1).

\section{Discussion}

In 2002, BMP was approved for use in the United States by the FDA for ALIF procedures, as an alternative to iliac crest-bone grafts (ICBG). ${ }^{8}$ Since then, studies have demonstrated that patients treated with BMP intraoperatively experience shorter operative duration, lower blood loss, shorter length of stay, lower reoperation rates, and faster returns to work than those treated with ICBG. ${ }^{14,16,18}$ Given the complexity of adult spinal deformity cases, achieving solid arthrodesis can be quite challenging. As a result, off-label use of BMP to improve outcomes for spinal deformity cases has been of particular interest to spine surgeons.

This study utilized a large national administrative dataset to determine the impact of intraoperative BMP on postoperative complications and revision rates in patients undergoing anterior cervical deformity surgery. Though the groups of patients receiving intraoperative BMP and not receiving BMP were well matched, it is important to note that there was a higher rate of tobacco use among those patients receiving BMP treatment, a finding that may be attributed to the idea that surgeons tend to administer adjunct BMP to patients at risk for 
non-union. Despite this imbalance, we found that the revision rate was consistently higher among patients who did not receive intraoperative BMP. However, certain postoperative complications were significantly more common among patients who received adjunct BMP.

\section{Postoperative Complications}

Though the initial industry-sponsored clinical trials evaluating the use of BMP in various common spine surgery procedures demonstrated nearly no side effects, several independent studies thereafter reported adverse effects of BMP use. ${ }^{20,26,27}$ In a study of 328,468 spinal fusion patients, BMP use was independently associated with increased the odds of any postoperative complication (OR $1.43,95 \%$ CI $1.1-1.7$ ). ${ }^{28}$ Another study described higher rates of heterotopic ossification, infection, dysphagia, hematoma formation, and neurological deficits to be associated with BMP use in non-FDA approved spinal procedures. ${ }^{29}$ However, these findings may vary by procedure type and surgical approach, as one study found an increased incidence of complications with BMP use in anterior cervical fusion, whereas there was no such increase for thoracolumbar or posterior cervical fusions. ${ }^{30}$ The fear of developing a postoperative complication following cervical deformity may be the driver of decreasing BMP use over time in anteriorly corrected patients described in this study. Our study also found an increased frequency of deficiency and posthemorrhagic anemia among patients who received BMP during surgery to correct cervical spinal deformity, though other complications previously reported in other procedures that utilized intraoperative BMP (e.g. dysphagia, deep vein thrombosis, wound infection) were not significantly more frequent. (Table 2) Further, these results are particularly interesting since surgeon use of BMP would be more common in more complex deformity cases, thus leading to a difference in patient selection in the groups.

\section{Revision Surgery}

Additionally, our study assessed the effect of use of BMP on the reoperation rate after cervical deformity surgery. Many previous clinical trials have demonstrated that BMP improves the likelihood of solid fusion, which could potentially decrease the need for revision surgery. ${ }^{9-12}$ However, the literature regarding this topic is inconclusive, with some studies finding decreased reoperation rates, and others identifying no such difference. ${ }^{25,31} \mathrm{We}$ discovered a significantly reduced revision surgery rate among patients treated with intraoperative BMP at 90 days postoperatively, and this effect persisted to 2 years postoperatively. Given the complexity of cervical spinal deformity cases, the risk for 
hardware failure or non-union is quite high. Thus, the ability of BMP to reduce revision by increasing chances of arthrodesis in such cases is significant. However, previous studies have demonstrated that the impact of BMP on arthrodesis is not achieved until at least 6 months postoperatively. ${ }^{8,32,33}$ As such, the benefit to 90 -day revision rates is likely due to other factors. For example, Jaeger and colleagues found that BMP had lower rates of instrumentation failure compared to autologous iliac crest bone graft for isolated L5-S1 ALIF. ${ }^{34}$ Considering all failures were identified by the 3 month follow-up visit in this study, it is possible that the decreased revision rate at early timepoint discovered in our study is due to decreased rate of hardware failure, whereas late benefits can be attributed to higher rates of arthrodesis. We hypothesized that the reduced revision rate would lower healthcare utilization, which has traditionally reached costs upward of $\$ 100,000$ postoperatively. ${ }^{35,36}$ However, our study found that total payments remained higher among patients who received intraoperative BMP up to 2 years after the index surgery (Table 3). Spine surgeons should use their judgement in administering BMP to cervical deformity patients, weighing increased likelihood of superior fixation against the likely development of postoperative complications and increased financial burden.

\section{Limitations}

The limitations of MarketScan analyses have been previously reported by this group. ${ }^{37-39}$ Due to the nature of the data available, potential questions and analyses are constrained by the specificity of the ICD-9 codes. For example, this study was not able to stratify by use of low vs high dose BMP. Furthermore, although only multilevel procedures were included in this study, we were unable to control for length of fusion, operative time, and blood loss -factors which can impact postoperative complication rates. BMP may be used in situations where non-union is a concern or the surgery is more complex, and these operative characteristics were unavailable to us. However, there were significant efforts made to ensure validity of the findings. First, a relative homogenous cohort was assembled using cervical spine deformity surgery CPT codes. Nevertheless, we believe this study provides useful insight into the efficacy and safety of BMP in the surgical management of cervical spinal deformity.

\section{Conclusion}

Although the use of intraoperative BMP has demonstrated benefits in fusion integrity in ACD surgery, concern should remain with its association with increased rates of postoperative 
complications. This study identified an increased rate of postoperative complications in patients who received BMP; however, this may be confounded by increased complexity of surgery. Spine surgeons should weigh these benefits and drawbacks in identify optimal candidates of BMP use in ACD surgery.

\section{References}

1. Emery SE. Cervical spondylotic myelopathy: diagnosis and treatment. JAAOSJournal of the American Academy of Orthopaedic Surgeons. 2001;9(6):376-388.

2. Tundo F, Avila MJ, Willard L, et al. Spinal alignment, surgery, and outcomes in cervical deformity: A practical guide to aid the spine surgeon. Clin Neurol Neurosurg. 2019;185:105496.

3. Yadla S, Maltenfort MG, Ratliff JK, Harrop JS. Adult scoliosis surgery outcomes: a systematic review. Neurosurg Focus. 2010;28(3):E3.

4. McDonnell MF, Glassman SD, Dimar JR, 2nd, Puno RM, Johnson JR. Perioperative complications of anterior procedures on the spine. J Bone Joint Surg Am. 1996;78(6):839-847.

5. Faciszewski T, Winter RB, Lonstein JE, Denis F, Johnson L. The surgical and medical perioperative complications of anterior spinal fusion surgery in the thoracic and lumbar spine in adults. A review of 1223 procedures. Spine (Phila Pa 1976). 1995;20(14):1592-1599.

6. Kim YJ, Bridwell KH, Lenke LG, Glattes CR, Rhim S, Cheh G. Proximal junctional kyphosis in adult spinal deformity after segmental posterior spinal instrumentation and fusion: minimum five-year follow-up. Spine (Phila Pa 1976). 2008;33(20):21792184.

7. Urist MR. Bone: formation by autoinduction. Science. 1965;150(3698):893-899.

8. Burkus JK, Transfeldt EE, Kitchel SH, Watkins RG, Balderston RA. Clinical and radiographic outcomes of anterior lumbar interbody fusion using recombinant human bone morphogenetic protein-2. Spine (Phila Pa 1976). 2002;27(21):2396-2408.

9. Boden SD, Kang J, Sandhu H, Heller JG. Use of recombinant human bone morphogenetic protein-2 to achieve posterolateral lumbar spine fusion in humans: a prospective, randomized clinical pilot trial: 2002 Volvo Award in clinical studies. Spine (Phila Pa 1976). 2002;27(23):2662-2673.

10. Dimar JR, Glassman SD, Burkus KJ, Carreon LY. Clinical outcomes and fusion success at 2 years of single-level instrumented posterolateral fusions with recombinant human bone morphogenetic protein-2/compression resistant matrix versus iliac crest bone graft. Spine (Phila Pa 1976). 2006;31(22):2534-2539; discussion 2540 .

11. Glassman SD, Dimar JR, Carreon LY, Campbell MJ, Puno RM, Johnson JR. Initial fusion rates with recombinant human bone morphogenetic protein-2/compression resistant matrix and a hydroxyapatite and tricalcium phosphate/collagen carrier in posterolateral spinal fusion. Spine (Phila Pa 1976). 2005;30(15):1694-1698.

12. Glassman SD, Carreon LY, Djurasovic M, et al. RhBMP-2 versus iliac crest bone graft for lumbar spine fusion: a randomized, controlled trial in patients over sixty years of age. Spine (Phila Pa 1976). 2008;33(26):2843-2849. 
13. Ong KL, Villarraga ML, Lau E, Carreon LY, Kurtz SM, Glassman SD. Off-label use of bone morphogenetic proteins in the United States using administrative data. Spine (Phila Pa 1976). 2010;35(19):1794-1800.

14. Burkus JK. Bone morphogenetic proteins in anterior lumbar interbody fusion: old techniques and new technologies. Invited submission from the Joint Section Meeting on Disorders of the Spine and Peripheral Nerves, March 2004. J Neurosurg Spine. 2004;1(3):254-260.

15. Carlisle E, Fischgrund JS. Bone morphogenetic proteins for spinal fusion. Spine J. 2005;5(6 Suppl):240s-249s.

16. Burkus JK, Sandhu HS, Gornet MF. Influence of rhBMP-2 on the healing patterns associated with allograft interbody constructs in comparison with autograft. Spine (Phila Pa 1976). 2006;31(7):775-781.

17. Boakye M, Mummaneni PV, Garrett M, Rodts G, Haid R. Anterior cervical discectomy and fusion involving a polyetheretherketone spacer and bone morphogenetic protein. J Neurosurg Spine. 2005;2(5):521-525.

18. Burkus JK, Heim SE, Gornet MF, Zdeblick TA. The effectiveness of rhBMP-2 in replacing autograft: an integrated analysis of three human spine studies. Orthopedics. 2004;27(7):723-728.

19. Carragee EJ, Hurwitz EL, Weiner BK. A critical review of recombinant human bone morphogenetic protein-2 trials in spinal surgery: emerging safety concerns and lessons learned. Spine J. 2011;11(6):471-491.

20. Fu R, Selph S, McDonagh M, et al. Effectiveness and harms of recombinant human bone morphogenetic protein- 2 in spine fusion: a systematic review and meta-analysis. Ann Intern Med. 2013;158(12):890-902.

21. Tannoury CA, An HS. Complications with the use of bone morphogenetic protein 2 (BMP-2) in spine surgery. Spine J. 2014;14(3):552-559.

22. Glassman SD, Gum JL, Crawford CH, 3rd, Shields CB, Carreon LY. Complications with recombinant human bone morphogenetic protein-2 in posterolateral spine fusion associated with a dural tear. Spine J. 2011;11(6):522-526.

23. Poorman GW, Jalai CM, Boniello A, Worley N, McClelland S, 3rd, Passias PG. Bone morphogenetic protein in adult spinal deformity surgery: a meta-analysis. Eur Spine J. 2017;26(8):2094-2102.

24. Puvanesarajah V, Jain A, Cancienne JM, et al. BMP Use and the Risk of Revision Surgery After Long Posterolateral Fusions in the Elderly. Clin Spine Surg. 2017;30(7):E931-e937.

25. Paul JC, Lonner BS, Vira S, Kaye ID, Errico TJ. Use of Recombinant Bone Morphogenetic Protein Is Associated With Reduced Risk of Reoperation After Spine Fusion for Adult Spinal Deformity. Spine (Phila Pa 1976). 2016;41(1):E15-21.

26. Smucker JD, Rhee JM, Singh K, Yoon ST, Heller JG. Increased swelling complications associated with off-label usage of rhBMP-2 in the anterior cervical spine. Spine (Phila Pa 1976). 2006;31(24):2813-2819.

27. Buttermann GR. Prospective nonrandomized comparison of an allograft with bone morphogenic protein versus an iliac-crest autograft in anterior cervical discectomy and fusion. Spine J. 2008;8(3):426-435.

28. Cahill KS, Chi JH, Day A, Claus EB. Prevalence, complications, and hospital charges associated with use of bone-morphogenetic proteins in spinal fusion procedures. Jama. 2009;302(1):58-66.

29. Epstein NE. Complications due to the use of BMP/INFUSE in spine surgery: The evidence continues to mount. Surg Neurol Int. 2013;4(Suppl 5):S343-352. 
30. Williams BJ, Smith JS, Fu KM, et al. Does bone morphogenetic protein increase the incidence of perioperative complications in spinal fusion? A comparison of 55,862 cases of spinal fusion with and without bone morphogenetic protein. Spine (Phila Pa 1976). 2011;36(20):1685-1691.

31. Deyo RA, Ching A, Matsen L, et al. Use of bone morphogenetic proteins in spinal fusion surgery for older adults with lumbar stenosis: trends, complications, repeat surgery, and charges. Spine (Phila Pa 1976). 2012;37(3):222-230.

32. McCarthy I, O'Brien M, Ames C, et al. Incremental cost-effectiveness of adult spinal deformity surgery: observed quality-adjusted life years with surgery compared with predicted quality-adjusted life years without surgery. Neurosurg Focus. 2014;36(5):E3.

33. McCarthy IM, Hostin RA, Ames CP, et al. Total hospital costs of surgical treatment for adult spinal deformity: an extended follow-up study. Spine J. 2014;14(10):23262333.

34. Varshneya K, Pendharkar AV, Azad TD, Ratliff JK, Veeravagu A. A Descriptive Analysis of Spinal Cord Arteriovenous Malformations: Clinical Features, Outcomes, and Trends in Management. World Neurosurg. 2019.

35. Azad TD, Vail D, Bentley J, et al. Initial Provider Specialty Is Associated With Longterm Opiate Use in Patients With Newly Diagnosed Low Back and Lower Extremity Pain. Spine (Phila Pa 1976). 2019;44(3):211-218.

36. Azad TD, Varshneya K, Ho AL, Veeravagu A, Sciubba DM, Ratliff JK.

Laminectomy Versus Corpectomy for Spinal Metastatic Disease-Complications, Costs, and Quality Outcomes. World Neurosurg. 2019.

Figure 1. BMP Use in Anterior Deformity Correction Over Time. The percentage of anterior deformity cases from 2007-2015 that involved intraoperative BMP use (y-axis) was plotted by year (x-axis).

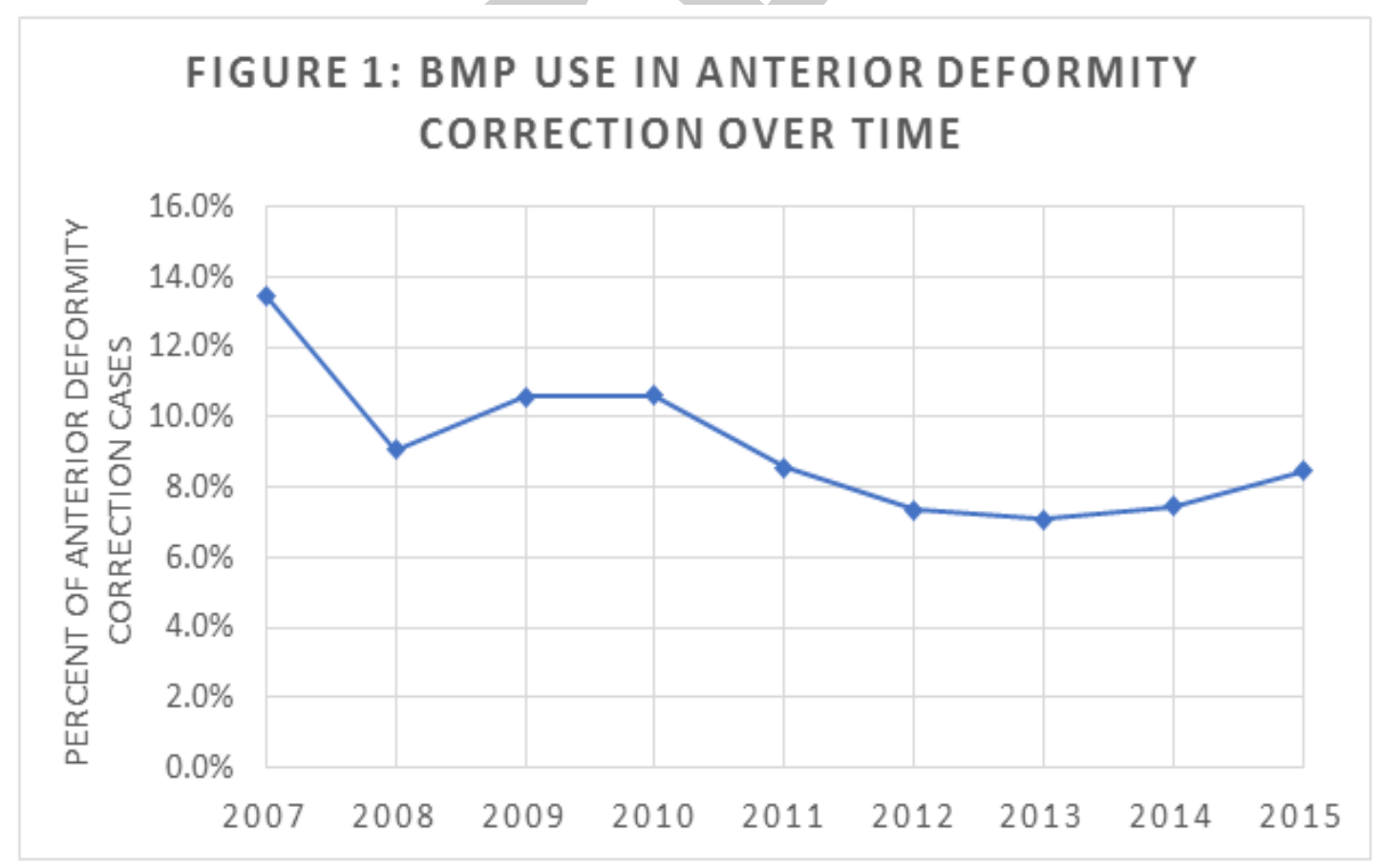


Table 1. Demographic and Clinical Factors

\begin{tabular}{|l|c|c|c|}
\hline \multicolumn{1}{|c|}{ Variables } & Non-BMP $\mathrm{n}=12394$ & BMP $\mathrm{n}=1155$ & P value \\
\hline Age mean (SD) & 51.5 & 52.1 & 0.0710 \\
\hline Female N (\%) & 63.7 & 63.6 & 0.5300 \\
\hline CHF N (\%) & 6.1 & 6.5 & 0.6093 \\
\hline Hypertension N (\%) & 41.1 & 43.6 & 0.0952 \\
\hline Tobacco N (\%) & 29.0 & 33.4 & 0.0015 \\
\hline Diabetes N (\%) & 20.1 & 19.1 & 0.4372 \\
\hline Liver Disease N (\%) & 7.2 & 6.6 & 0.4135 \\
\hline Cardiac Arrythmias N (\%) & 15.7 & 15.8 & 0.8926 \\
\hline Osteoporosis N (\%) & 19.3 & 20.5 & 0.3193 \\
\hline Approach Type & & & $<0.0001$ \\
\hline \multicolumn{1}{|c|}{ Anterior } & 84.7 & 89.2 & \\
\hline Posterior & 9.0 & 7.7 & \\
\hline Circumferential & 6.2 & 2.1 & \\
\hline
\end{tabular}

Table 2. 90 Day Overall Complications

\begin{tabular}{|l|c|c|c|}
\hline \multicolumn{1}{|c|}{ Variables (\%) } & Non-BMP $\mathrm{n}=12394$ & BMP $\mathrm{n}=1155$ & P value \\
\hline Any complication & 27.6 & 31.1 & 0.0127 \\
\hline Dysphagia & 2 & 1.4 & 0.1669 \\
\hline Deep Vein Thrombosis & 0.5 & 0.4 & 0.8669 \\
\hline Deficiency Anemia & 9 & 11.6 & 0.0034 \\
\hline Hematoma & 1.7 & 2.3 & 0.1597 \\
\hline Posthemorrhagic Anemia & 11.9 & 14.7 & 0.005 \\
\hline Wound Dehiscence & 0.9 & 0.6 & 0.3731 \\
\hline Wound Infection & 1.9 & 2.5 & 0.1483 \\
\hline Stroke & 1 & 1.6 & 0.0803 \\
\hline PE & 1 & 0.9 & 0.7136 \\
\hline
\end{tabular}


Table 3. Quality Outcomes

\begin{tabular}{|c|c|c|c|}
\hline Variables & Non-BMP $n=12394$ & $\mathrm{BMP} n=1155$ & $P$ value \\
\hline Length of stay mean (SD) & 3.7 & 4 & 0.0213 \\
\hline \multicolumn{4}{|l|}{ Reoperations } \\
\hline 90 days & 28.3 & 14.5 & $<0.0001$ \\
\hline 180 days & 28.6 & 14.9 & $<0.0001$ \\
\hline 365 days & 29.2 & 15.7 & $<0.0001$ \\
\hline 730 days & 29.9 & 16.5 & $<0.0001$ \\
\hline \multicolumn{4}{|l|}{ Index Hospitalization Costs (mean) } \\
\hline Physician Payments & $\$ 10,680$ & $\$ 9,791$ & 0.0796 \\
\hline Hospital Payments & $\$ 62,532$ & $\$ 74,895$ & $<0.0001$ \\
\hline Total Payments & $\$ 80,211$ & $\$ 91,488$ & $<0.0001$ \\
\hline \multicolumn{4}{|c|}{ Total Payments (Index + Post Discharge) } \\
\hline 90 days & $\$ 85,838$ & $\$ 97,917$ & $<0.0001$ \\
\hline 365 days & $\$ 92,357$ & $\$ 103,270$ & 0.0004 \\
\hline 730 days & $\$ 97,620$ & $\$ 107,975$ & 0.0021 \\
\hline
\end{tabular}

Table 4. Multivariate Adjusted Analysis to Identify Impact of BMP on Outcomes

\begin{tabular}{|c|c|c|c|}
\hline \multicolumn{1}{|c|}{ Variables } & OR & $95 \% \mathrm{Cl}$ & $\mathrm{P}$ value \\
\hline Any complication & 1.22 & $1.07-1.40$ & $\mathbf{0 . 0 0 2 5}$ \\
\hline Reoperations & & & \\
\hline 90 days & 0.44 & $0.38-0.53$ & $<\mathbf{0 . 0 0 0 1}$ \\
\hline 180 days & 0.46 & $0.38-0.54$ & $<\mathbf{0 . 0 0 0 1}$ \\
\hline 365 days & 0.47 & $0.40-0.56$ & $<\mathbf{0 . 0 0 0 1}$ \\
\hline 730 days & 0.49 & $0.41-0.57$ & $<\mathbf{0 . 0 0 0 1}$ \\
\hline
\end{tabular}

